\title{
AVICENNA'S DOCTRINE AND INSTITUTIONAL REVIEW BOARD IN INTERNATIONAL BIO-MEDICAL RESEARCH
}

\author{
Amir Radfar ${ }^{1}$, Chekameh Kazerouninia ${ }^{2}$, Irina Filip ${ }^{3}$, Diana Patriche ${ }^{4}$, Seyed Ahmad Asgharzadeh Ahmadi ${ }^{5}$ \\ ${ }^{1}$ University of Massachusetts, Lowell, USA \\ 2University of Phoenix, Arizona, USA \\ ${ }^{3}$ University of Massachusetts, Amherst, USA \\ ${ }^{4}$ University of Medicine Alexandru loan Cuza, Bucharest, Romania \\ ${ }^{5}$ Babol University of Medical Sciences, Babol, Iran
}

\begin{abstract}
SUMMARY
Avicenna, an outstanding Persian physician and philosopher (980 AD-1037 AD), established a clinical treaty, or doctrine, without which medical experimentation would not have progressed. This doctrine emphasizes the ultimate divine power of God or a higher being over healing and mandates the patients' well-being as the crucial aspect in all medical care and experiments. The Institutional Review Board, as the ethical body that oversees clinical research, is in line with this doctrine. However, the lack of a homogenous and internationally recognized code of ethics, the decentralized work of ethics oversight committees, the improper implementation of established ethical standards and a shortage of scientific auditing capacities have raised concerns over the possible exploitation of vulnerable populations.
\end{abstract}

Key words: Avicenna, bioethics, IRB, Belmont report, vulnerable population, international research

Address for correspondence: A. Radfar, University of Massachusetts, Lowell, PO Box 17584, Encino, California, 91416, USA. E-mail: aradf001@ fiu.edu

Avicenna, a Persian physician scientist and philosopher (980 AD-1037 AD) and a pioneer of clinical trials, suggested several blueprints for the assessment of safety and the effects of medicine on patients. Avicenna's highly influential Canon of Medicine continues to inspire his modern colleagues. His clear understanding of pilot studies for drug quality, investigations into action time and reproducibility of treatment effects antedates modern clinical trials by one millennium (1).

Avicenna established a philosophy that perceives God as the ultimate healer and patients' well-being as the crucial aspect in all medical care and experiments. Avicenna's clinical treaties recall Hippocratic decorum but emphasize two ideas that are not in the Hippocratic tradition: the ultimate power of God over life, death, and healing and the obligation to care for the needy and preserve their rights. In the Canon of Medicine, his masterpiece, he highlights the facts that care for the sick requires empathy and an understanding of their rights.

Mandating care for those in need and observing the rights of vulnerable populations are aspects of the modern concept of the Institutional Review Board (IRB). Although there is no doubt that clinical trials are the crucial aspect of medicine to combat diseases, incidences of abuse in the name of research require independent oversight by ethical committees.

One of the most horrific of these incidents, which is often cited in medical history, is the Tuskegee, Alabama syphilis study (1932-1972). This incident has increased awareness of the need to protect vulnerable populations. The Belmont Report conceptualized the aim of the IRB as ensuring that all experiments follow the ethical principles that govern the protection of all human subjects in research (2).

Fischer et al. describe the IRB as "an independent reviewing body that evaluates investigational protocols prior to their implementation to ensure their validity and ethical integrity by implementing good clinical practices and standards" (3). In the United States, the IRB for biomedical sciences is composed of representatives from different areas of a hospital or medical university, including clinicians, ethicists, clergy, scientists and educators, who review all of the research proposals to safeguard and protect the human subjects involved in research projects.

The IRB ideally follows all of the basic ethical principles that govern the protection of human subjects (4). The research project cannot begin until the investigator addresses potential issues, and the contingent responses should be approved by the IRB.

IRBs place special emphasis on reviewing studies that involve vulnerable population groups, such as members of minority ethnic groups, children, the poor, women, soldiers, prisoners, international populations, and persons with impaired decision-making capacities. Eckenwiler defines vulnerability in terms of "threats to self-development, self-determination, and equality that exist independent of research" (5).

However, controversies over the functions of IRBs pose challenges to the implementation of modern clinical trials in developing countries. Empirical evidence indicates that patients' rights, the behaviour of health care providers and pharmaceutical innovation with regard to the burden of diseases differ in developing and developed countries. For instance, new medicines based 
on clinical trials in developing countries are not affordable by the people of those countries.

It is not uncommon for researchers to recruit human subjects from developing countries. In addition to many legitimate and ethical clinical trials in developing countries, the existence of health disparities and low socio-economic status often make the most vulnerable members of society targets of unethical clinical trials. Factors such as cost reduction and subject vulnerability, the leniency of laws and regulations, and a lack of established ethical guidelines in these countries make them ideal sites for undesirable or unnecessary clinical trials.

The lack of a homogenous and internationally recognized code of ethics, the decentralized work of ethics oversight committees, the improper implementation of established ethical standards and a shortage of scientific auditing capacities have raised concerns over the possible exploitation of vulnerable populations. Review boards have no way of controlling and determining the ethical clearance process for these vulnerable human subjects, who either suffer from terminal diseases or are desperate to obtain appropriate healthcare.

The existence of corruption, incompetence, dependency, bureaucracy, and the iron law of oligarchy can affect the decisionmaking process in ethical reviews. In cases of unethical clinical trial, IRB members and researchers find themselves managing conflicts of interest in situations of dual loyalty. The oversight mechanism, the functioning of ethics committees and the decisions of regulatory bodies are influenced by the interests of stakeholders, governments, researchers, academic and non-academic institutions, and industry. These decisions are expressed through uneven power relations among stakeholders, between stakeholders and IRBs, between IRBs and researchers, between sponsors and researcher and so on (6). As such, the research agendas of these clinical trials are influenced by financial incentives to researchers and/or institutions by those who are powerful enough to shape these agendas.

In conclusion, the role of IRBs in protecting vulnerable populations and the need to ensure that scientific ethics are not compromised through use of a global and centralized code of ethics have become crucial issues. Conducting unethical clinical trials by neglecting the health of vulnerable populations in the pursuit of benefits is a violation of human rights. Global efforts must be made to guarantee that the implementation of clinical trials in developing countries is relevant to the needs of these populations. As implied in Avicenna's doctrine, it is imperative for the biomedical research community and consumers to ensure that no patients' rights violations occur during the course of clinical trials.

Avicenna's legacy, the Canon of Medicine, opens with an eloquent treatise on the place of medicine within the human community. Medicine, says Avicenna, can be seen in three ways: as the pursuit of the practical art rewarded by money, as the practice of devotion to one's needy fellow humans, rewarded by gratitude from those who have experienced the physician's generosity, and, finally, as devotion to God, which has a dual reward: the good of the patient who is cured and the good of the physician who, touched by divine love, is granted insight into the realities of nature.

The biomedical research community must decide how and to what extent it is willing to learn and benefit from this great pioneer of ancient medicine for the betterment of modern medical experimentation.

\section{Conflict of interest}

None declared

\section{REFERENCES}

1. Colgan R. Advice to the young physician: on the art of medicine. New York: Springer Verlag; 2009.

2. U.S. Department of Health \& Human Services. The Belmont Report. Ethical Principles and Guidelines for the Protection of Human Subjects of Research [Internet]. The National Commission for the Protection of Human Subjects of Biomedical and Behavioral Research; 1979 [cited 2012 Oct 08]. Available from: http://www.hhs.gov/ohrp/humansubjects/ guidance/belmont.html.

3. Fischer C, Oneto C. Kaplan medical USMLE medical ethics: the 100 cases you are most likely to see on the exam. New York: Kaplan Publishing; 2006.

4. Coleman $\mathrm{CH}$, Bouësseau MC. How do we know that research ethics committees are really working? The neglected role of outcomes assessment in research ethics review. BMC Med Ethics. 2008 Mar 28;9:6.

5. Eckenwiler L. Moral reasoning and the review of research involving human subjects. Kennedy Inst Ethics J. 2001 Mar;11(1):37-69.

6. London L. Ethical oversight of public health research: can rules and IRBs make a difference in developing countries? Am J Public Health. 2002 Jul;92(7):1079-84. 